\title{
INVERSE MULTIPARAMETER EIGENVALUE PROBLEMS FOR MATRICES III
}

\author{
by PATRICK J. BROWNE* and B. D. SLEEMAN
}

(Received 25th August 1986)

\section{Introduction}

This note will complement and, in a certain sense, complete our earlier studies $[3,4]$ of the theory of inverse multiparameter eigenvalue problems for matrices. In those papers, we considered the so called "additive inverse problem" which, briefly stated for the 2-parameter case, asks for conditions on given $n \times n$ matrices $A, B, C$ and on given points $\left(s_{i}, t_{i}\right) \in \mathbb{R}^{2}, 1 \leqq i \leqq n$, under which a diagonal matrix $D$ can be found so that the 2-parameter eigenvalue problem

$$
(A+D+\lambda B+\mu C) x=0, \quad x \neq 0,
$$

can be solved when $(\lambda, \mu)=\left(s^{i}, t^{i}\right), 1=i=n$. Put another way, we look for conditions ensuring that the points $\left(s_{i}, t_{i}\right), 1 \leqq i \leqq n$, belong to the eigenvalues of (1.1).

Here we shall be concerned with the multiplicative inverse problem wherein we now seek conditions on the given $n \times n$ matrices $A, B, C$ and on the points $\left(s_{i}, t_{i}\right) \in \mathbb{R}^{2}$, $1 \leqq i \leqq n$, so that a diagonal matrix $D$ with positive entries can be found for which the 2-parameter eigenvalue problem

$$
\left(D^{1 / 2} A D^{1 / 2}+\lambda B+\mu C\right) x=0, \quad x \neq 0,
$$

has $\left(s_{i}, t_{i}\right), 1 \leqq i \leqq n$, on its eigencurves.

Our main result (Theorem 2.3) states that if the points $\left(s_{i}, t_{i}\right)$ are cone-ordered (Hypothesis 2.1) and satisfy a certain "spacing" condition (Hypothesis 2.2) then such a diagonal matrix $D$ exists.

As in $[3,4]$, our motivation comes from the work of Hadeler $[5,6,7]$ who studied inverse problems for the classical 1-parameter case $A x=\lambda x$. A short list of references for this field can be found in [3]. We shall follow the ideas of [4] and use topological degree theory as our main tool. Section 2 contains our central result and we close with some remarks about standard applications to linked systems of multiparameter problems and to quadratic eigenvalue problems.

*The research of this author was supported in part by SERC grant GR/D/88724. 


\section{One equation with two parameters}

Suppose we are given $n \times n$ Hermitean matrices $A, B, C$ where, without loss of generality, we assumed that the diagonal elements of $A$ satisfy $a_{i i}=1,1 \leqq i \leqq n$. For each $(\lambda, \mu) \in \mathbb{R}^{2}$, the matrix

$$
W(\lambda, \mu)=A+\lambda B+\mu C
$$

is also Hermitean with eigenvalues

$$
\rho_{1}(\lambda, \mu) \leqq \cdots \leqq \rho_{n}(\lambda, \mu) .
$$

We are interested in the eigencurves defined by

$$
Z_{i}(A)=\left\{(\lambda, \mu) \in \mathbb{R}^{2} \mid \rho_{i}(\lambda, \mu)=0\right\}, \quad 1 \leqq i \leqq n .
$$

As we pointed out in [3], it will be enough for us here to assume that one of $B, C$ is positive or negative definite to ensure $Z_{i}(A) \neq \phi, 1 \leqq i \leqq n$, although various weaker conditions would suffice, cf. [1]. We need the cone $K \subset \mathbb{R}^{2}$ given by

$$
K=\left\{(\lambda, \mu) \in \mathbb{R}^{2} \mid \lambda(B x, x)+\mu(C x, x) \leqq 0, \quad \forall x \in \mathbb{C}^{n}\right\},
$$

and impose the following conditions.

Hypothesis 2.1. The points $\left(s_{i}, t_{i}\right)$ satisfy

$$
\left(s_{1}, t_{1}\right) \in K^{0} \equiv \operatorname{int}(K),-s_{i} b_{i i}-t_{i} c_{i i}>0, \quad 1 \leqq i \leqq n,
$$

and are $K$-ordered, i.e.

$$
\left(s_{j}, t_{j}\right)-\left(s_{i}, t_{i}\right) \in K^{0} \quad \text { whenever } \quad j \geqq i .
$$

The necessity for an assumption concerning the ordering of these points was discussed in $[3,4]$.

Next, given real numbers $x_{i} \geqq 0,1 \leqq i \leqq n$, we define

$$
g_{i}^{j}(x)=\sum_{\substack{k=1 \\ k \neq i}}^{n}\left|x_{i}^{1 / 2} a_{i k} x_{k}^{1 / 2}+s_{j} b_{i k}+t_{j} c_{i k}\right|, \quad 1 \leqq i, \quad j \leqq n .
$$

From now on we shall use

$$
x=\left(x_{1}, \ldots, x_{n}\right), x_{i}=-s_{i} b_{i i}-t_{i} c_{i i}, \quad 1 \leqq i \leqq n .
$$

Hypothesis 2.2.

$$
\left(s_{j}-s_{i}\right) b_{i i}+\left(t_{j}-t_{i}\right) c_{i i}<-g_{i}^{j}(x)-2 g_{j}^{j}(x)-g_{i}^{i}(x)
$$




$$
\begin{array}{r}
\left(s_{k}-s_{j}\right) b_{k k}+\left(t_{k}-t_{j}\right) c_{k k}<-g_{k}^{j}(x)-2 g_{j}^{j}(x)-g_{k}^{k}(x) \\
1 \leqq i<j<k \leqq n .
\end{array}
$$

Note that Hypothesis 2.1 ensures that the left hand sides of these two inequalities are non-positive. Now select $\eta>0$ and consider the open bounded region $E \subset \mathbb{R}^{n}$ given by

$$
\begin{aligned}
& E=\{v=\left(v_{1}, \ldots, v_{n}\right) \in \mathbb{R}^{n} \mid v_{i}>0, \\
& v_{1}+s_{1} b_{11}+t_{1} c_{11}>-\eta, v_{n}+s_{n} b_{n n}+t_{n} c_{n n}<\eta, \\
& v_{i}+s_{j} b_{i i}+t_{j} c_{i i}+g_{i}^{j}(v)<v_{j}+s_{j} b_{j j}+t_{j} c_{j j}-g_{j}^{j}(v) \\
& v_{j}+s_{j} b_{j j}+t_{j} c_{j j}+g_{j}^{j}(v)<v_{k}+s_{j} b_{k k}+t_{j} c_{k k}-g_{k}^{j}(v) \\
&1 \leqq i<j<k \leqq n\} .
\end{aligned}
$$

It is easy to check that $x \in E$.

For $v \in E$ we write $D(v)=\operatorname{diag}\left(v_{1}, \ldots, v_{n}\right)$. Also we put $\hat{B}=\operatorname{diag}\left(b_{11}, \ldots, b_{n n}\right), \hat{C}=$ $\operatorname{diag}\left(c_{11}, \ldots, c_{n n}\right)$ and $\breve{A}=A-I, \breve{B}=B-\hat{B}, \breve{C}=C-\hat{C}$. If $0 \leqq \theta \leqq 1$ we list the eigenvalues of the matrix

$$
M_{\theta}^{j}(v)=\theta\left[D(v)^{1 / 2} \check{A} D(v)^{1 / 2}+s_{j} \check{B}+t_{j} \check{C}\right]+D(v)+s_{j} \hat{B}+t_{j} \hat{C}
$$

in increasing order as

$$
\omega_{1}^{j}(\theta, v) \leqq \cdots \leqq \omega_{n}^{j}(\theta, v), \quad 1 \leqq j \leqq n .
$$

Consider the mapping $F_{\theta}: E \rightarrow \mathbb{R}^{n}$ given by

$$
F_{\theta}(v)=\left(\omega_{1}^{1}(\theta, v), \ldots, \omega_{n}^{n}(\theta, v)\right) .
$$

The problem of finding a diagonal matrix $D$ so that $\left(s_{i}, t_{i}\right) \in Z_{i}\left(D^{1 / 2} A D^{1 / 2}\right), 1 \leqq i \leqq n$, is equivalent to finding a point $v \in E$ for which $F_{1}(v)=0$.

For $v \in E$ we see that

$$
\begin{array}{r}
v_{i}+s_{j} b_{i i}+t_{j} c_{i i}<v_{j}+s_{j} b_{j j}+t_{j} c_{j j}<v_{k}+s_{j} b_{k k}+t_{j} c_{k k}, \\
1 \leqq i<j<k \leqq n,
\end{array}
$$

and so it follows that

$$
F_{0}(v)=v-x
$$

Thus $F_{0}(v)=0$ has a unique solution $v=x$. Moreover, in terms of the topological degree we have, see Lloyd [8],

$$
d\left(F_{0}, E, 0\right)=1
$$


Clearly, $F_{0}$ and $F_{1}$ are homotopy equivalent and so to use the homotopy equivalence of topological degree we need to show that for each $\theta \in[0,1]$, we have $0 \notin F_{\theta}(\partial E)$. Accordingly, suppose $v \in \partial E$ and $F_{\theta}(v)=0$. Should $v \in \partial E$ by virtue of $v_{1}+s_{1} b_{11}+t_{1} c_{11}=-\eta$, then $M_{\theta}^{1}(v)$ is positive semidefinite since its smallest eigenvalue is zero. Thus $v_{1}+s_{1} b_{11}+$ $t_{1} c_{11} \geqq 0-a$ contradiction. In like fashion we can discuss the case $v_{n}+s_{n} b_{n n}+t_{n} c_{n n}=\eta$. Should $v_{i}=0$ for some $i$, we again use the positive semi-definiteness of $M_{\theta}^{1}(v)$ to infer that $s_{1} b_{i i}+t_{1} c_{i i} \geqq 0$ which contradicts Hypothesis 2.1 .

Next we note that the matrix $M_{\theta}^{j}(v)$ has diagonal entries $v_{i}+s_{j} b_{i i}+t_{j} c_{i i}, 1 \leqq i \leqq n$, which are the centres of the Gersgorin circles for this matrix. The radii of the circles are $\theta g_{i}^{j}(v)$ respectively. From the relations defining $E$ we see that the circles corresponding to $i=1, \ldots, j-1$ are all disjoint from and lie to the left of the $j$ th circle which in turn is disjoint from the circles corresponding to $i=j+1, \ldots, n$ which lie to the right of the $j$ th circle. Using the theorems of Hadamard and Gersgorin [2, Theorems 6.2.1, 6.2.2, p. 231] we claim that the $j$ th circle contains $\omega_{j}^{i}(\theta, v)$ so that if $F_{\theta}(v)=0$ we must have

$$
\left|v_{j}+s_{j} b_{j j}+t_{j} c_{j j}\right| \leqq \theta g_{j}^{j}(v)
$$

This observation is sufficient to complete the proof that

$$
F_{\theta}(v) \neq 0 \text { for } v \in \partial E \text {. }
$$

The upshot of our remarks is

Theorem 2.3. Under Hypotheses 2.1, 2.2 there is a diagonal $D=\operatorname{diag}\left(v_{1}, \ldots, v_{n}\right)$ with $v_{i}>0,1 \leqq i \leqq n$, such that $\left(s_{i}, t_{i}\right) \in Z_{i}\left(D^{1 / 2} A D^{1 / 2}\right)$ and

$$
\left|v_{i}+s_{i} b_{i i}+t_{i} c_{i i}\right| \leqq g_{i}^{i}(v), \quad 1 \leqq i \leqq n .
$$

As in $[4,7]$ we can also claim

Theorem 2.4. The conclusion of Theorem 2.3. holds if equality is permitted in Hypothesis 2.2.

It is instructive to consider the case $C=0, B=-I$. This generates a 1-parameter theorem which parallels although does not exactly coincide with Hadeler's result [7, Theorem 3].

The cone $K$ becomes equivalent to the non-negative half-line $\mathbb{R}+$ and our ordering hypotheses reduce to $s_{n} \geqq s_{n-1} \ldots \geqq s_{1}>0$. The quantities $g_{i}^{j}(x)$ are now given by

$$
g_{i}^{j}(x)=x_{i}^{1 / 2} \sum_{k \neq i}\left|a_{i k} x_{k}^{1 / 2}\right|,
$$

i.e. they are $j$-independent. Hypothesis 2.2 reads

$$
s_{j}-s_{i} \leqq-2\left(g_{i}^{i}+g_{j}^{j}\right), \quad 1 \leqq i<j \leqq n,
$$


and under these conditions we conclude the existence of $D$ such that the eigenvalues of $D^{1 / 2} A D^{1 / 2}$ are $s_{1}, \ldots, s_{n}$. We note that the eigenvalues of $D A$ will then also be $s_{1}, \ldots, s_{n}$.

In $[3,4]$ we applied the theorems on inverse problems for one equation in two (or more) spectral parameters to problems involving linked systems of such equations and to quadratic eigenvalue problems. The apllications are immediate and straightforward and it will suffice for us to note here that similar results can be based upon the theorem in this note. We leave details to the reader.

\section{REFERENCES}

1. P. Binding and P. J. Browne, Spectral properties of two-parameter eigenvalue problems, Proc. Roy. Soc. Edinburgh 89A (1981), 157-173.

2. E. K. Buum, Numerical Analysis and Computation, Theory and Practice (Addison-Wesley, Reading, Mass. 1972).

3. P. J. Browne and B. D. Sleeman, Inverse multiparameter eigenvalue problems for matrices, Proc. Roy. Soc. Edinburgh 100A (1985), 29-38.

4. P. J. Browne and B. D. Sleeman, Inverse multiparameter eigenvalue problems for matrices, II, Proc. Edinburgh Math. Soc. 29 (1986), 343-348.

5. K. P. Hadeler, Ein inverses eigenwert problem, Linear Algebra Appl. 1 (1968), 83-101.

6. K. P. HADELER, Multiplicative inverse eigenvert probleme, Linear Algebra Appl. 2 (1969), 6586.

7. K. P. Hadeler, Existenz- und eindeutigkeitssätze für engenwertaufgaben mit hilfe des topologischen abbildungsgrades, Arch. Rat. Mech. Anal. 42 (1971), 317-322.

8. N. G. Lloyd, Degree Theory (Cambridge University Press, 1978).

Department of Mathematics and Statistics

UNIVERSITY OF CALGARY

CALGaRY

Alberta

Canada T2N IN4
Department of Mathematical SCiences UNIVERSITY OF DUNDEE DUNDEE DDI 4HN

SCOTLAND 